\title{
A systematic review and meta-analysis of traditional Chinese medicine with chemotherapy in breast cancer
}

\author{
Gang Shi ${ }^{1 \#}$, Dan Yu ${ }^{1 \#}$, Juan Wu ${ }^{1 \#}$, Yanru Liu ${ }^{2}$, Ruizhen Huang ${ }^{3}$, Cheng Shun Zhang ${ }^{4}$ \\ ${ }^{1}$ Department of Pharmacy Service, Hospital of Chengdu University of Traditional Chinese Medicine, Chengdu, China; ${ }^{2}$ Department of \\ Cardiovascular, Chengdu Qingbajiang District Traditional Chinese Medicine Hospital, Chengdu, China; ${ }^{3}$ Department of Cardiovascular, Hospital \\ of Chengdu University of Traditional Chinese Medicine, Chengdu, China; ${ }^{4}$ Acupuncture and Tuina School/Third Teaching Hospital, Chengdu \\ University of Traditional Chinese Medicine, Chengdu, China \\ Contributions: (I) Conception and design: G Shi, D Yu, J Wu, CS Zhang; (II) Administrative support: R Huang; (III) Provision of study materials or \\ patients: G Shi, D Yu, J Wu, Y Liu, CS Zhang; (IV) Collection and assembly of data: All authors; (V) Data analysis and interpretation: G Shi, D Yu, J \\ Wu, Y Liu, R Huang; (VI) Manuscript writing: All authors; (VII) Final approval of manuscript: All authors. \\ "These authors contributed equally to this work. \\ Correspondence to: Ruizhen Huang. Hospital of Chengdu University of Traditional Chinese Medicine, No. 39 Shi-er-qiao Road, Chengdu 610072, \\ China. Email: zhenzhen41@163.com; Cheng Shun Zhang. Chengdu University of Traditional Chinese Medicine, No. 37 Shi-er-qiao Road, Chengdu, \\ China. Email: chengshun.zhang@cdutcm.edu.cn.
}

Background With the continuous progression of a new generation of adjuvant chemotherapy, the survival time of breast cancer patients has also been significantly improved. Chemotherapy alone will cause a series of side effects, which will seriously affect the quality of life of breast cancer patients. Chinese medicine combined with neoadjuvant chemotherapy has a unique advantage in the treatment of breast cancer.

Methods: English databases were searched using combinations of the following search terms: "traditional Chinese medicine", "neoadjuvant hemotherapy", "breast cancer", and "tumor of breast". Publications in which traditional Chinese medicine (TCM) combined with neoadjuvant therapy was the experimental group and chemotherapy alone was the control group were screened.

Results: A total of 12 publications were included in the meta-analysis. The efficiency of the performance status score was used to test for heterogeneity, $\mathrm{Chi}^{2}=2.95, \mathrm{df}=5, \mathrm{P}=0.71>0.1, \mathrm{I}^{2}=0 \%, \mathrm{Z}=3.36$, odds ratio (OR) $=2.61$, and $95 \%$ confidence interval (CI), 1.49-4.58. The results of the heterogeneity test of the effective rate of the objective curative effect were as follows: $\mathrm{Chi}^{2}=1.04, \mathrm{df}=7, \mathrm{P}=0.99>0.1, \mathrm{I}^{2}=0 \%<50 \%, \mathrm{Z}=2.42$, OR $=2.00$, and $95 \%$ CI, 1.14-3.49. The results for the heterogeneity test of the TCM syndrome score were as follows: $\mathrm{I}^{2}=83 \%, \mathrm{P}<0.00001$, mean difference $(\mathrm{MD})=8.84,95 \% \mathrm{CI}, 6.43-11.25, \mathrm{P}<0.05$. The results for the heterogeneity test of the incidence of adverse reactions in the digestive system after chemotherapy were as follows: $\mathrm{Chi}^{2}=1.15, \mathrm{df}=8, \mathrm{P}=1.00>0.1, \mathrm{I}^{2}=0 \%<50 \%, \mathrm{Z}=1.68$, OR $=0.04,95 \% \mathrm{CI},-0.01$ to 0.09 .

Discussion: The meta-analysis confirmed that using TCM combined with neoadjuvant chemotherapy to treat breast cancer has obvious advantages over chemotherapy alone in terms of the objective curative effect, the performance status score effective rate, the TCM syndrome score change, and the incidence of gastrointestinal adverse reactions after chemotherapy.

Keywords: Traditional Chinese medicine (TCM); neoadjuvant chemotherapy; breast cancer

Submitted Apr 07, 2021. Accepted for publication May 18, 2021.

doi: $10.21037 /$ gs-21-284

View this article at: http://dx.doi.org/10.21037/gs-21-284 


\section{Introduction}

Breast cancer is a common malignant tumor among women in China, and its annual incidence continues to rise (1). There are approximately 280,000 new cases of breast cancer each year, and breast cancer is the number one killer of women (2). Over time, chemotherapy has become the main method for the comprehensive treatment of breast cancer, and neoadjuvant chemotherapy has made rapid progress (3). In recent years, the combined application of taxanes and anthracyclines and chemotherapy in traditional Chinese medicine (TCM) has prolonged the survival time of breast cancer patients. Anthracyclines mainly include aclacinomycin, epirubicin, daunorubicin, and taxeria drugs, which constitute the cornerstone of modern breast cancer chemotherapy (4). If only neoadjuvant chemotherapy is used alone, the toxic and side effects of patients will be relatively large, which will affect the patients' living standards to a large extent.

TCM is a unique medical resource in China, and it occupies a pivotal position in China's medical and health system (5). A large number of clinical studies have suggested that TCM combined with neoadjuvant chemotherapy has good therapeutic effects on breast cancer. Specifically, it has been suggested that TCM relieves the symptoms of patients, reduces the side effects of chemotherapy, and improves the quality of life of patients. However, large, randomized, prospective, and placebo-controlled studies are lacking (6). Chen et al. (7) noted that there were some problems in clinical trials of TCM combined with neoadjuvant chemotherapy. As the reliability of the research results are insufficient, TCM treatments of breast cancer cannot be promoted internationally. We searched for relevant articles that had been published since the establishment of the databases, and found that there are two main treatment methods for treating breast cancer with TCM: (I) oral TCM treatments, which mainly use "qi"-tonifying agents, such as Shengmai San; and (II) intravenous drip treatments, including Shengmai and Canmai injections (8). For patients undergoing chemotherapy, the basic situation is complicated, oral TCM is often used, and the patient's overall condition should be considered (9).

Research on the application of TCM combined with neoadjuvant chemotherapy to breast cancer has gradually developed. However, whether it can improve the therapeutic effect and reduce the toxic and side effects, the results of different studies are not uniform. In this study, the clinical efficacy of Chinese medicine combined with neoadjuvant treatment of breast cancer was evaluated through systematic evaluation and meta-analysis of the clinical real data of breast cancer patients. A comprehensive understanding of the clinical efficacy of breast cancer was carried out, exploring its current problems. It was hoped to provide effective basis for literature research and authoritative treatment and promote the international development of Chinese medicine.

We present the following article in accordance with the PRISMA reporting checklist (available at http://dx.doi. org/10.21037/gs-21-284).

\section{Methods}

\section{Literature search}

The PubMed, Medline, CMCC, Embase, Chinese Biomedical Literature Database, China National Knowledge Network (CNKI) database, Wanfang database, VIP database, and Baidu Academic database were searched. The search period ran from the establishment of the databases to December 25, 2020. Composite logic retrieval and Boolean logic retrieval were used to select the relevant publications. Chinese databases were searched using combinations of the following search terms: "traditional Chinese medicine", "neoadjuvant chemotherapy", "breast cancer", and "breast tumor". English databases were searched using combinations of the following search terms: "traditional Chinese medicine", "neoadjuvant hemotherapy", "breast cancer", and "tumor of breast". All search terms were combined freely. After many searches, search engines were used to locate the publications. Experts and researchers in the field were contacted to ensure we were up to date with the latest research progress.

\section{Literature inclusion and exclusion criteria}

Publications were included in the meta-analysis if they met the following inclusion criteria: (I) the study was a clinical randomized controlled trial (RCT) of TCM combined with neoadjuvant chemotherapy to treat breast cancer and was published before December 25, 2020; (II) the general data of the test group and the control group were well balanced and comparable; (III) the experimental group was treated with TCM combined with neoadjuvant chemotherapy, and the control group was treated with chemotherapy alone; (IV) for the pathological control analysis, the index comparison was reliable in the $95 \%$ confidence interval $(\mathrm{CI})$; and $(\mathrm{V})$ 
the diagnosis of breast cancer was based on the standards of the World Health Organization.

Publications were excluded from the meta-analysis if they met any of the following exclusion criteria: (I) the study was not an RCT; (II) the treatment method of TCM was acupuncture and moxibustion (a non-oral drug treatment); (III) numerous articles had been published on the study; (IV) animal tests or breast tumor cell tests were undertaken; and/ or $(\mathrm{V})$ complete data could not be obtained for the study by contacting the original author.

Two senior experts independently screened each abstract and the full text of the publication, and conducted 3 preliminary experiments before screening. If the views of the 2 experts differed, a consensus was reached through discussion, or a third expert was invited to arbitrate.

\section{Clinical evaluation indicators of breast cancer}

The clinical evaluation indicators of breast cancer include the effective rate of the performance status score, the effective rate of the objective curative effect, the TCM syndrome score change, and the incidence of an adverse digestive system after chemotherapy. Specifically:

Effective rate of physical state $=$ number of affected cases of

physical state $\div$ total number of cases

Objective response rate $=($ partial response + complete response $) \div$ total response

\section{Data extraction}

The 2 experts used a unified Excel table to extract data independently; 3 preliminary experiments were undertaken before extraction. If the views of the 2 experts differed, a consensus was reached through discussion, or a third expert was invited to arbitrate. The data extracted for the included research included: (I) the title of the included publication; (II) the first author and publication year; (III) the name of the publication; (IV) the date when the research was published; (V) general information about the research subjects, such as the average age, gender, body mass index (BMI), and disease course; (VI) the therapeutic effect comparison between the experimental group and the control group.

\section{Risk of bias assessment}

The risk of bias was assessed by the 2 experts at the same time. If the 2 experts disagreed, a consensus was reached through discussion, or a third expert was asked to arbitrate. In this study, the Cochrane Collaboration tool was used for the "bias risk assessment" of the RCTs, and Rev Man 5.3 was used to evaluate the quality of the publications. The evaluation criteria included the generation of a random sequence, the blind method, the hiding of the allocation scheme, the integrity of the data results, and the research results. The above 5 aspects were judged as "high-risk bias", "low-risk bias", or "unclear".

\section{Literature quality assessment}

The risk of bias was assessed by 2 experts at the same time. If the 2 experts disagreed, a consensus was reached through discussion, or a third expert was asked to arbitrate. In this study, the JADAD scale of the Cochrane Collaboration was used for the quality grading. The specific JADAD quality grading method is shown in Table 1. A JADAD score of 4-7 indicates a high-quality study (with low-risk bias), and a score of $0-3$ indicates a low-quality study (with high-risk bias).

\section{Statistical methods}

Rev Man 5.3 was used for the statistical analysis. Odds ratios (ORs) and 95\% CIs were used to evaluate the efficiency of the performance status score, the effective rate of the objective curative effect, the change of the TCM syndrome score, and the incidence of an adverse digestive system after chemotherapy. The Rev Man 5.3's risk of bias assessment chart was used to assess the risk bias of the included publications. Each effect was expressed using a 95\% CI. If the heterogeneity test showed that $\mathrm{P}>0.1$ and $\mathrm{I}^{2}<50 \%$, a fixed-effects model was used for the meta-analysis. If the heterogeneity test showed that $\mathrm{P}<0.1$ and $\mathrm{I}^{2}>50 \%$, a random-effects model was used for the meta-analysis.

\section{Results}

\section{Results and basic information of the included publications}

In total, 1,685 publications were screened; 911 publications were eliminated by reading the abstracts and titles, and articels with replication of study subjects $(n=485)$, study type other than randomized controlled trial $(\mathrm{n}=259)$, and study subject other than breast cancer $(\mathrm{n}=18)$ were excluded (see Figure 1). Figure 2 shows the JADAD quality grading results. Two publications $(10,11)$ received scores of 4-7 and 
Table 1 JADAD rating scale

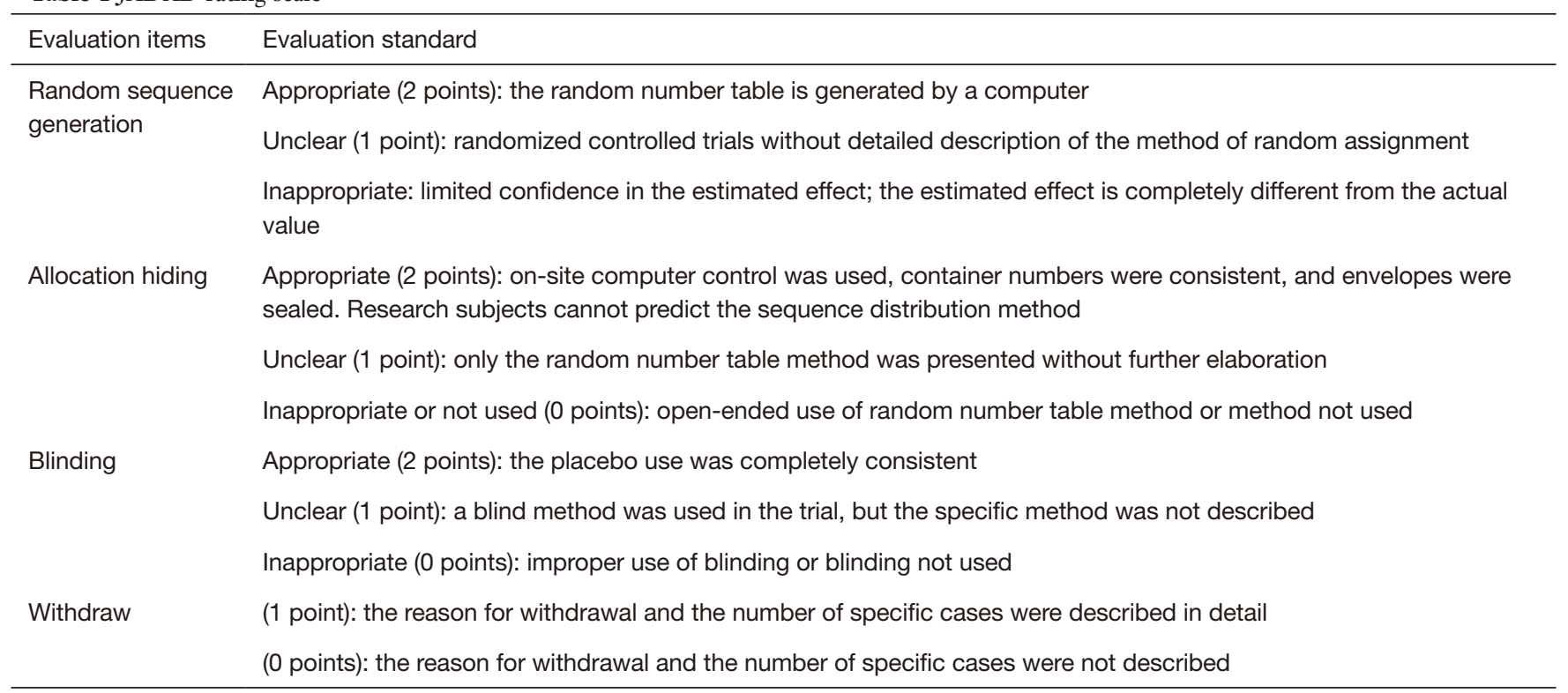

$\mathrm{RCT}$, randomized controlled trial.

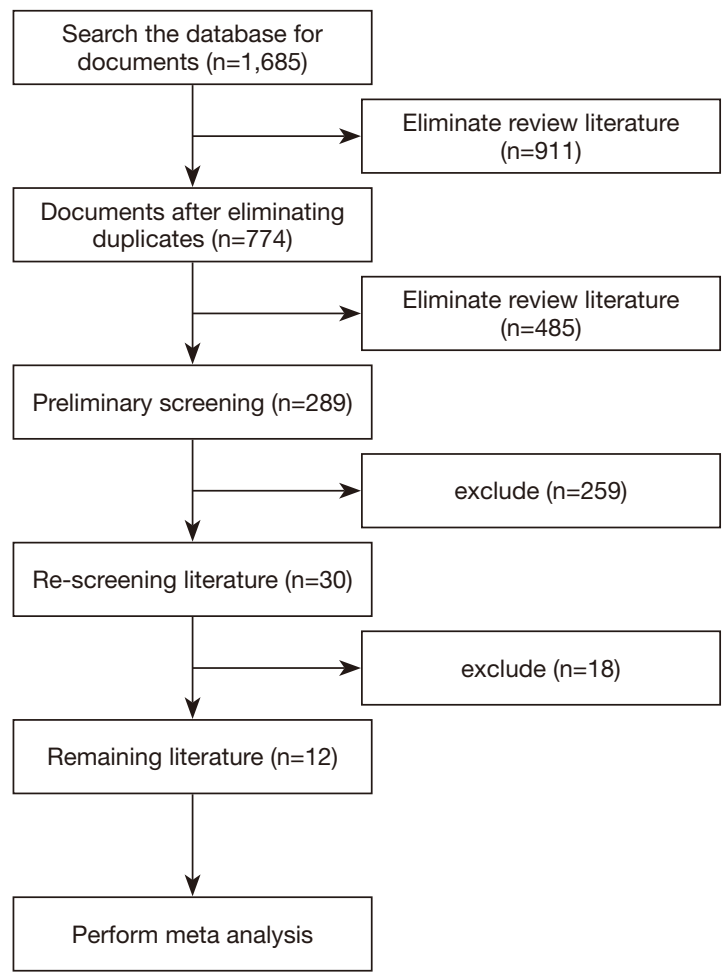

Figure 1 Search flow chart.
10 received scores of $0-3$.

Twelve publications met the inclusion criteria, and there were 534 patient cases, including 8 in the chemotherapy control group. All of the 12 publications used small sample sizes ranging from 49 to 92 , and the study subjects were all aged over 18. The 12 publications described the gender, age, BMI, and disease course of patients in the experimental and control groups in detail. The basic information of the research subjects is set out in Table 2.

\section{The results of the risk bias evaluations of publications}

Figures 3 and 4 show the results of multiple risk bias evaluations of the publications produced by Rev Man 5.3. Among the 12 RCTs included in this work, 3 (12-14) described the correct random allocation method, $2(15,16)$ described the correct random allocation method and the concealment of the allocation plan in detail, and 1 (17) was evaluated by the blind method; the other articles did not use the blind method. The measurement indicators in this work were laboratory indicators determined by a computer. Therefore, it was deemed that all the studies used the blind 
method correctly.

\section{The efficiency of the performance status score}

A total of 6 publications analyzed the effectiveness of performance status scoring in RCT experiments. There were 462 cases in total $(231$ cases in the experimental group, and 231 cases in the control group). An overall heterogeneity test was performed $\left(\mathrm{Chi}^{2}=2.95, \mathrm{df}=5\right.$, $\left.\mathrm{P}=0.71>0.1, \mathrm{I}^{2}=0 \%<50 \%\right)$. The combined effect-size test showed that $\mathrm{Z}=3.36$.

Based on an analysis of the above results, the 12 publications included in the meta-analysis were homogeneous, and the combined effect size used a fixedeffects model $(\mathrm{OR}=2.61,95 \% \mathrm{CI}, 1.49-4.58)$. A

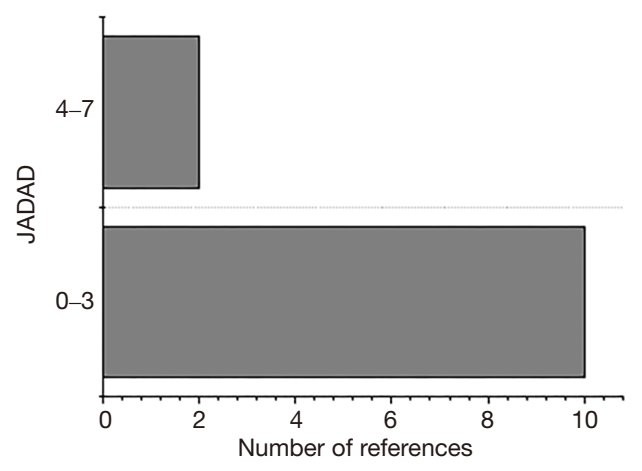

Figure 2 JADAD quality classification results. combination of TCM and neoadjuvant chemotherapy appeared to have obvious advantages over the treatment of chemotherapy alone in improving patients' performance status scores (see Figure 5). The horizontal line of the 95\% $\mathrm{CI}$ of most publications fell on the right side of the invalid vertical line, and that of a few studies crossed the invalid vertical line. A fixed-effects model was adopted for the overall analysis; the difference between the experimental group and the control group was not statistically significant.

Rev Man 5.3 was used to produce the performance status scoring efficiency funnel chart (see Figure 6). The circles of some publications were basically symmetrical to the midline, which indicates that the research accuracy was high and there was no bias in the publications.

\section{Effectiveness of objective curative effect}

A total of 8 publications analyzed the effectiveness of the objective curative effects in the RCTs. There were 485 cases in total (246 in the experimental group, and 239 in the control group). An overall heterogeneity test was performed $\left(\mathrm{Chi}^{2}=1.04, \mathrm{df}=7, \mathrm{P}=0.99>0.1, \mathrm{I}^{2}=0 \%<50 \%\right)$. The combined effect-size test showed that $\mathrm{Z}=2.42$.

Based on the analysis of the above results, the included 12 publications were homogeneous, and the combined effect size was analyzed using the fixed-effects model (OR $=2.00,95 \%$ CI, 1.14-3.49). The results showed that the

Table 2 General information about research subjects in the included publications

\begin{tabular}{lccccccc}
\hline Author & Year & Cases & Age (years old $)$ & Male & Female & BMl $\left(\mathrm{m}^{2} / \mathrm{kg}\right)$ & Course of disease $(\mathrm{year})$ \\
\hline Ji & 2020 & 49 & $40.4 \pm 9.7$ & 28 & 21 & $28.7 \pm 5.0$ & $5.0 \pm 2.1$ \\
Liew & 2019 & 68 & $61.6 \pm 9.9$ & 32 & 36 & $30.9 \pm 3.9$ & $3.2 \pm 2.1$ \\
Li & 2018 & 52 & $42.8 \pm 8.5$ & 25 & 27 & $30.6 \pm 5.3$ & $5 \pm 1.6$ \\
Chen & 2018 & 86 & $56.0 \pm 8.6$ & 41 & 45 & $27.3 \pm 4.5$ & $9.1 \pm 5.8$ \\
Wang & 2015 & 56 & $45.3 \pm 5.8$ & 27 & 29 & $27.4 \pm 3.2$ & $4.5 \pm 2.3$ \\
Wong & 2014 & 48 & $25.7 \pm 6.5$ & 25 & 23 & $22.6 \pm 2.4$ & $4.6 \pm 2.8$ \\
Kim & 2015 & 76 & $63.1 \pm 7.3$ & 32 & 44 & $31.62 \pm 5.4$ & $5.3 \pm 1.1$ \\
Cohen & 2002 & 92 & $45.8 \pm 6.5$ & 45 & 47 & $25.6 \pm 4.3$ & $4.5 \pm 2.3$ \\
Liu & 2016 & 64 & $54.3 \pm 9.7$ & 28 & 26 & $24.5 \pm 4.0$ & $5.2 \pm 1.5$ \\
Lee & 2014 & 91 & $49.6 \pm 8.6$ & 42 & 49 & $23.9 \pm 2.3$ & $5.2 \pm 2.5$ \\
Meng & 2017 & 50 & $52.3 \pm 8.3$ & 23 & 27 & $26.5 \pm 4.6$ & $4.5 \pm 2.3$ \\
Zhang & 2020 & 54 & $69.6 \pm 10.8$ & 26 & 28 & $27.4 \pm 5.0$ & $3.5 \pm 0.3$ \\
\hline
\end{tabular}




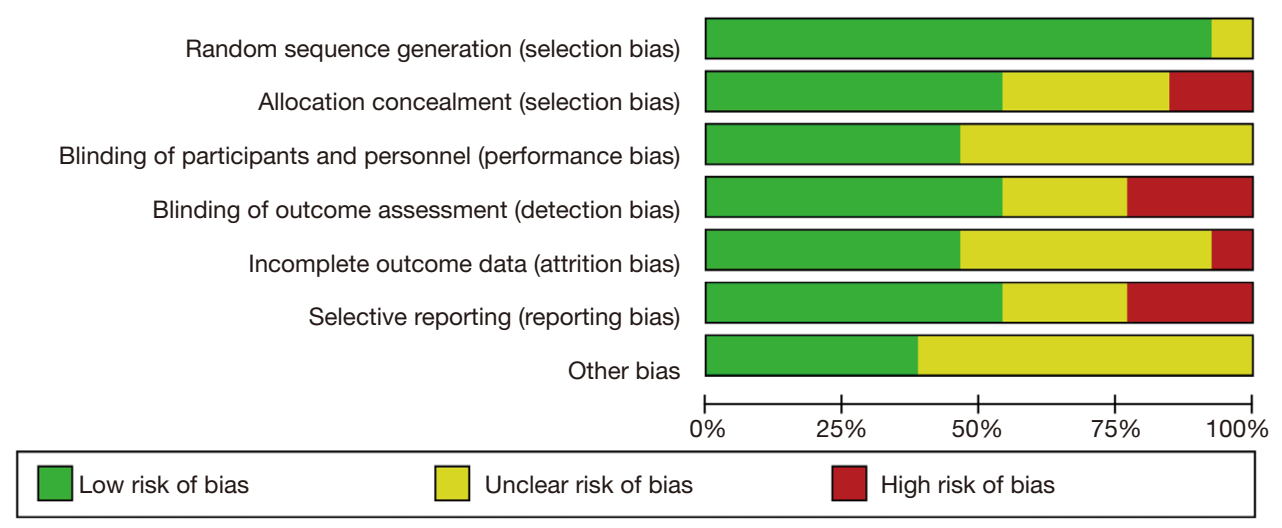

Figure 3 Publication risk bias-evaluation results.

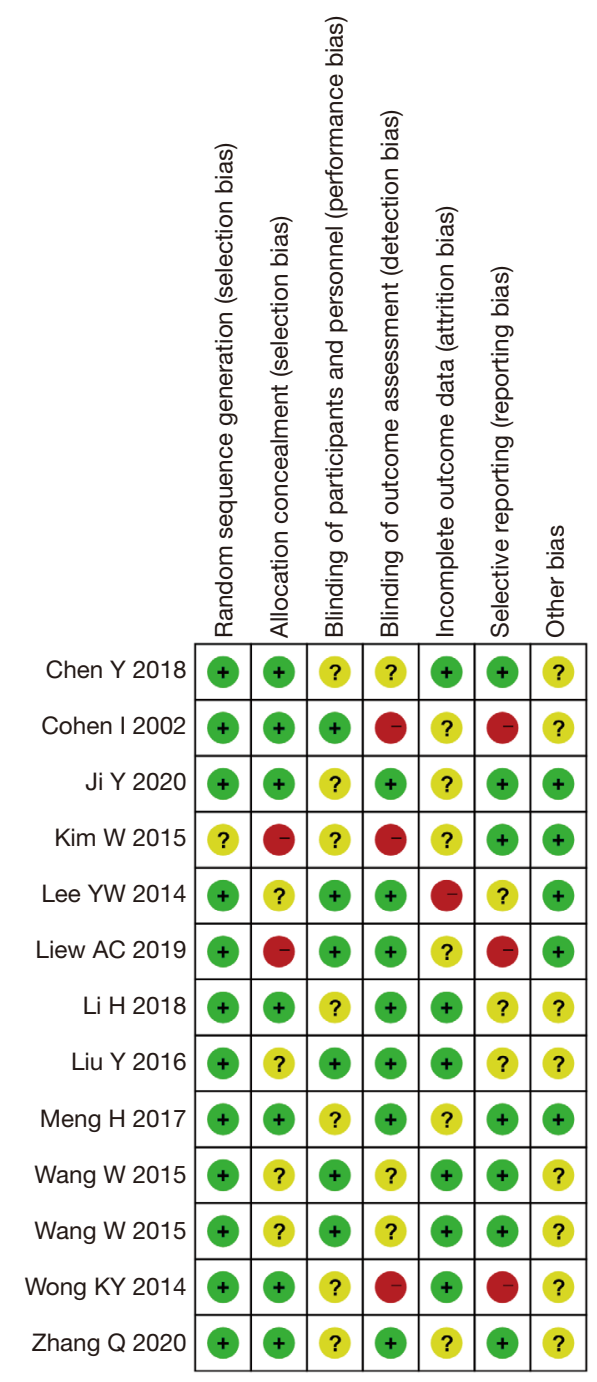

Figure 4 Bias-evaluation results of the included publications in relation to multiple risks. combination of TCM and neoadjuvant chemotherapy had obvious advantages over the treatment of chemotherapy alone in improving the objective curative effect (see Figure 7). The horizontal lines in the $95 \%$ CI of most publications were invalid, and the vertical lines were crossed. A fixed-effects model was used for the metaanalysis; the difference between the experimental group and the control group was not statistically significant.

Rev Man 5.3 was used to produce the objective curative effects funnel chart (see Figure 8). The circles of some publications were basically symmetrical to the midline, which indicates that the research accuracy was high and there was no bias the in publications.

\section{Changes in TCM syndrome scores}

A total of 8 publications analyzed the changes in TCM syndrome scores in RCTs. There were 539 cases in total (271 cases in the experimental group, and 268 cases in the control group). The overall heterogeneity test showed that $\mathrm{I}^{2}=83 \%(\mathrm{P}<0.00001)$, and the heterogeneity between each experimental group was relatively large. The horizontal line of the $95 \%$ CI of most publications fell to the right of the invalid vertical line. A random-effects model was used for the meta-analysis; the difference between the experimental group and the control group was statistically significant. The results showed that TCM combined with neoadjuvant chemotherapy improved the pathological symptoms of breast cancer in TCM (mean difference (MD) $=8.84,95 \%$ CI, 6.43-11.25, $\mathrm{P}<0.05$; Figure 9).

Rev Man 5.3 was used to produce the TCM syndrome scores funnel chart (see Figure 10). The circles of some 


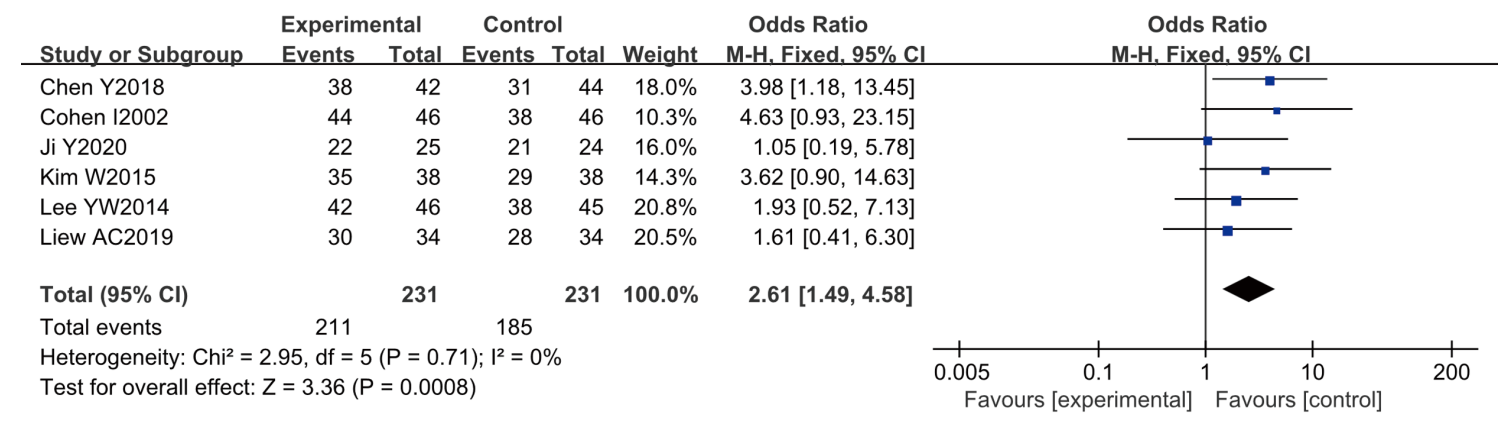

Figure 5 Forest plot of the efficiency of the performance status score.

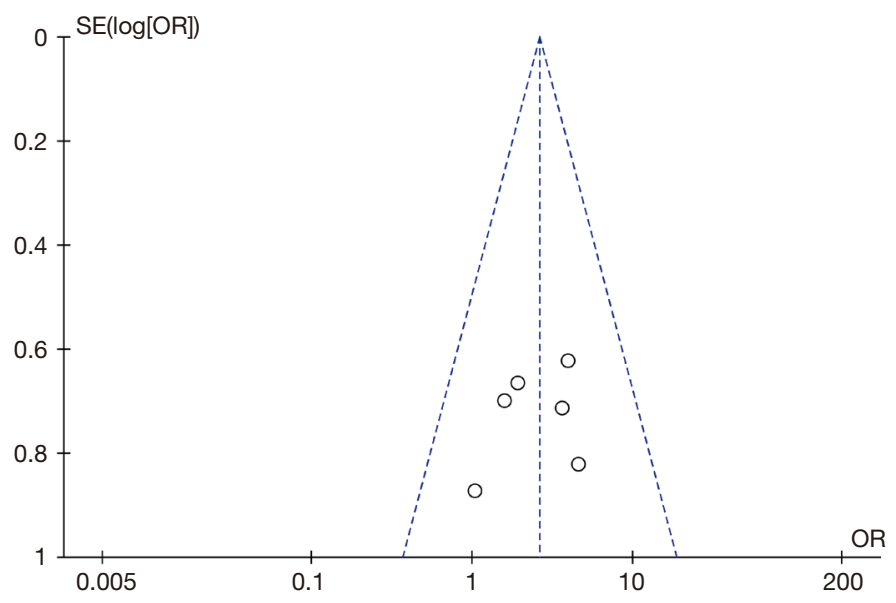

Figure 6 Funnel chart of the efficiency of the performance status score.

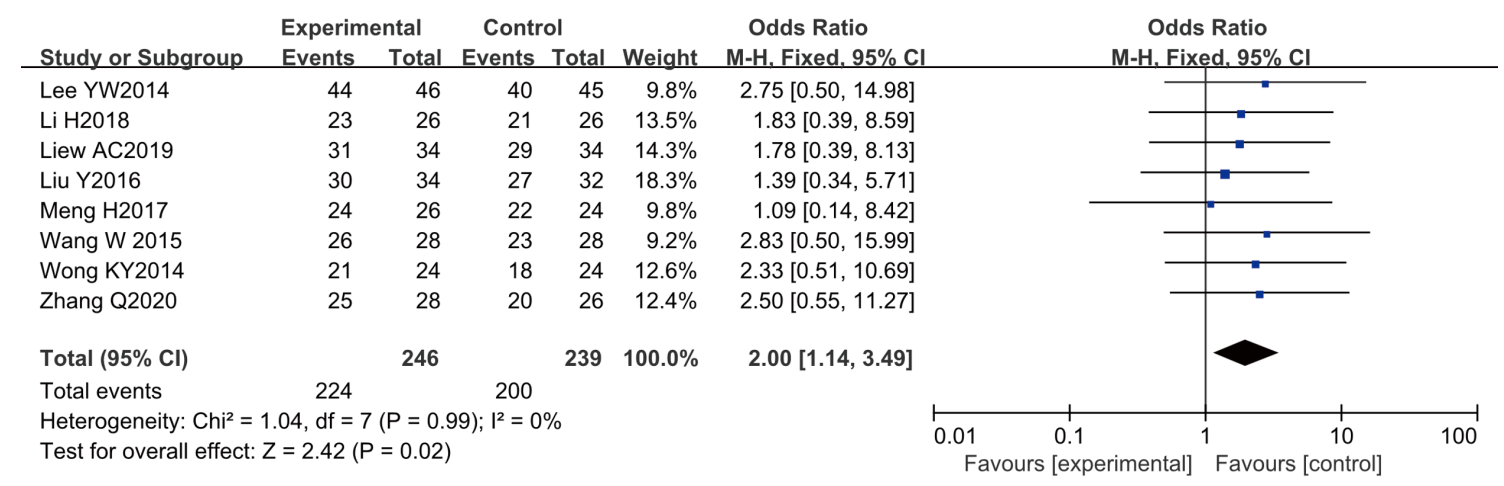

Figure 7 Forest plot of the objective curative effect.

publications were concentrated on the center line and were basically symmetrical to the center line, which indicates that the research accuracy was high and there was no bias in the publications.

\section{Incidence of adverse digestive system reactions after chemotherapy}

A total of 9 publications analyzed the incidence of adverse 


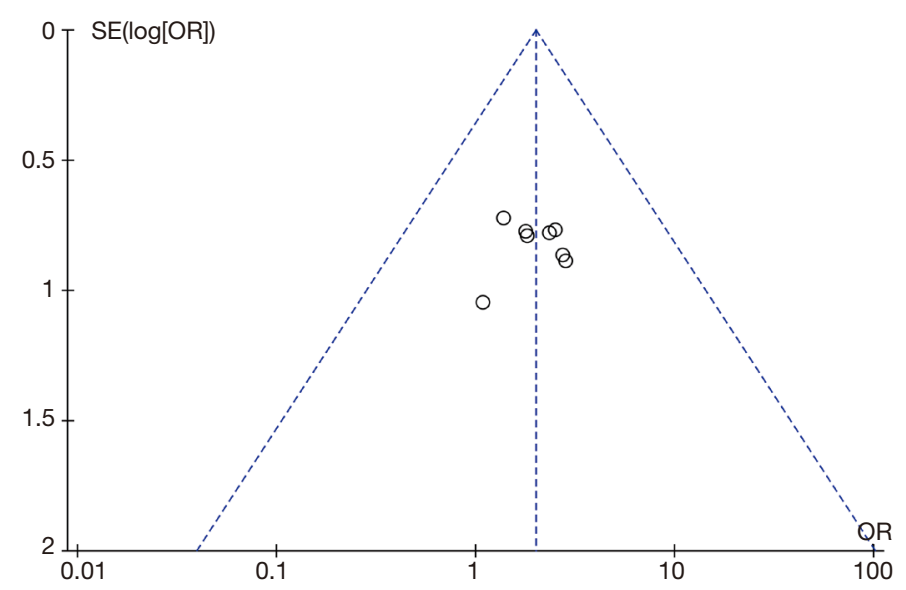

Figure 8 Funnel chart of the objective curative effect.

\begin{tabular}{|c|c|c|c|c|c|c|c|c|c|c|c|}
\hline \multirow{3}{*}{ Study or Subgroup } & \multicolumn{3}{|c|}{ Experimental } & \multicolumn{2}{|c|}{ Control } & \multirow{2}{*}{\multicolumn{2}{|c|}{ Iotal Weight }} & \multirow{3}{*}{$\begin{array}{l}\text { Mean Difference } \\
\text { IV. Random, } 95 \% \mathrm{Cl}\end{array}$} & \multirow{2}{*}{\multicolumn{3}{|c|}{$\begin{array}{c}\text { Mean Difference } \\
\text { IV. Random. } 95 \% \mathrm{Cl}\end{array}$}} \\
\hline & Mean & SD $T$ & Total & Mean & SD $T$ & & & & & & \\
\hline & 41 & 8 & 42 & 30 & 6 & 44 & $12.3 \%$ & & & $\sigma$ & \\
\hline Cohen 12002 & 40 & 9 & 46 & 26 & 5 & 46 & $12.3 \%$ & $14.00[11.02,16.98]$ & & $=$ & \\
\hline Ji Y2020 & 24 & 5 & 25 & 18 & 4 & 24 & $13.0 \%$ & $6.00[3.47,8.53]$ & & $\pi$ & \\
\hline Kim W2015 & 35 & 6 & 38 & 25 & 7 & 38 & $12.4 \%$ & $10.00[7.07,12.93]$ & & $\rightarrow$ & \\
\hline Li H2018 & 22 & 4 & 26 & 19 & 6 & 26 & $12.6 \%$ & $3.00[0.23,5.77]$ & & - & \\
\hline Liew AC2019 & 31 & 8 & 34 & 22 & 5 & 34 & $12.0 \%$ & $9.00[5.83,12.17]$ & & - & \\
\hline Liu Y2016 & 32 & 7 & 34 & 21 & 4 & 32 & $12.7 \%$ & $11.00[8.27,13.73]$ & & $=$ & \\
\hline Meng H2017 & 24 & 5 & 26 & 17 & 5 & 24 & $12.6 \%$ & $7.00[4.23,9.77]$ & & - & \\
\hline Total $(95 \% \mathrm{Cl})$ & & & 271 & & & 268 & $100.0 \%$ & $8.84[6.43,11.25]$ & & $\bullet$ & \\
\hline $\begin{array}{l}\text { Heterogeneity: } \mathrm{Tau}^{2}= \\
\text { Test for overall effect }\end{array}$ & $\begin{array}{l}9.94 ; C h i \\
Z=7.19\end{array}$ & & $\begin{array}{l}\text { D2, df = } \\
00001)\end{array}$ & & & & $=83 \%$ & & $\begin{array}{cc}-50 & -25 \\
\text { Favours [experimental] }\end{array}$ & Favours ${ }^{2}$ & $\begin{array}{l}25 \\
\text { control] }\end{array}$ \\
\hline
\end{tabular}

Figure 9 Forest plot of TCM syndrome score changes. TCM, traditional Chinese medicine.

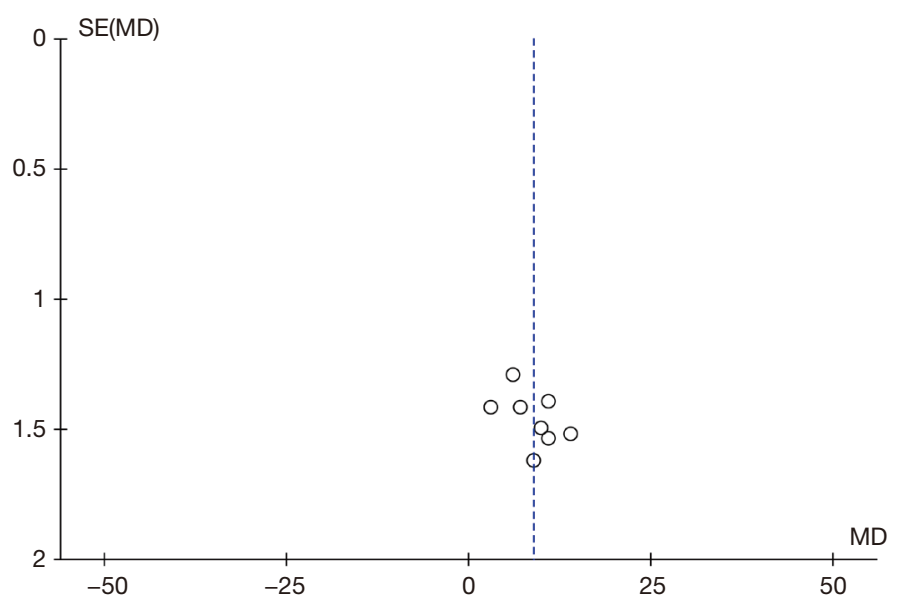

Figure 10 Funnel chart of TCM syndrome integral changes. TCM, traditional Chinese medicine. 


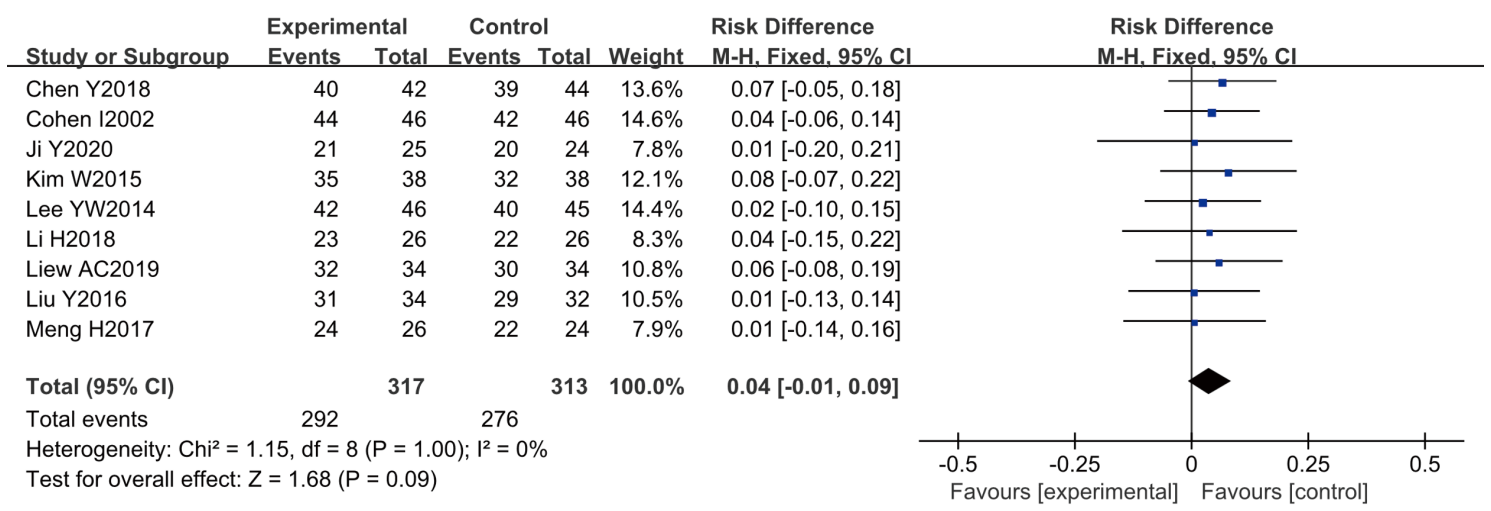

Figure 11 Forest plot of the incidence of adverse digestive system reactions after chemotherapy.

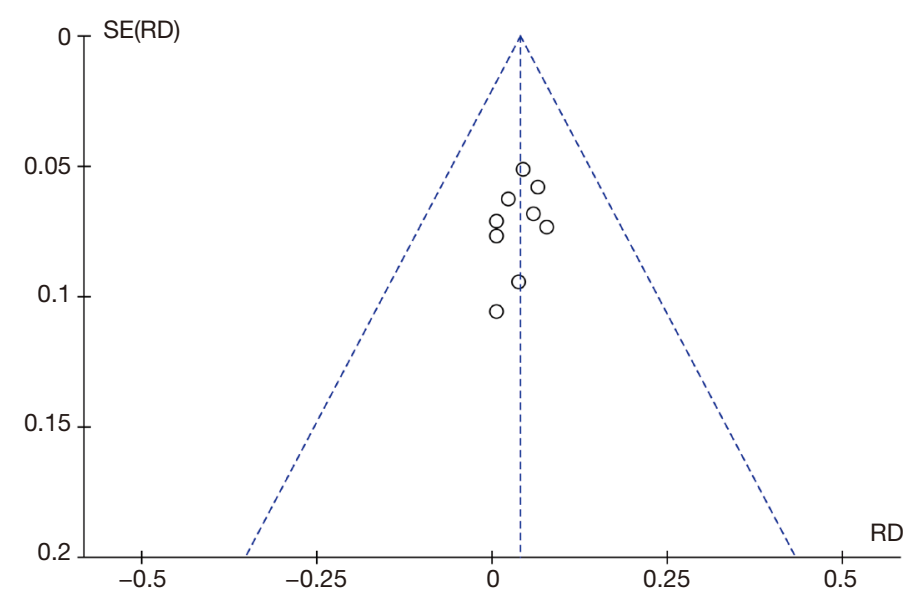

Figure 12 Funnel chart of the incidence of adverse digestive system reactions after chemotherapy.

reactions in the digestive system after chemotherapy in RCTs. There were 630 cases in total (317 in the experimental group, and 313 in the control group). An overall heterogeneity test was performed $\left(\mathrm{Chi}^{2}=1.15, \mathrm{df}=8\right.$, $\left.\mathrm{P}=1.00>0.1, \mathrm{I}^{2}=0 \%<50 \%\right)$. The combined effect-size test showed that $\mathrm{Z}=1.68$.

Based on the analysis of the above results, the included 9 publications were homogeneous, and the combined effect size used a fixed-effects model (OR $=0.04,95 \% \mathrm{CI}$, -0.01 to 0.09 ). The results showed that TCM combined with neoadjuvant chemotherapy had a notable advantage over the treatment of chemotherapy alone in reducing the incidence of adverse reactions in the digestive system after chemotherapy (see Figure 11). The horizontal lines in the 95\% CI of most publications were invalid, and the vertical lines crossed. A fixed-effects model was adopted for the meta-analysis; the difference between the experimental group and the control group was not statistically significant.

Rev Man 5.3 was used to produce the funnel chart of the incidence of adverse reactions in the digestive system after chemotherapy (see Figure 12). The circles of some publications were basically symmetrical to the midline, which indicates that the research accuracy was high and there was no bias in the publications.

\section{Discussion}

TCM occupies a very important position in the treatment of cancer in China, which is a kind of special treatment way in China. As China gradually moves towards internationalization, the use of TCM to treat tumors has received international medical attention. However, clinical research of TCM in oncology is relatively limited. Despite the obvious curative effect of this breast cancer treatment, 
there remains a lack of multicentered trials, randomized trials, and double-blind-related studies. The lack of high quality and insufficient persuasive evidence in the literature studies lead to the failure of the internationalization of the scientific research on the treatment of tumor by TCM. Clinical workers in developed countries usually collect relevant literatures of high-quality small-sample randomized controlled trials and conduct evaluation through meta-analysis, and the theoretical results are widely accepted.

Twelve publications were included in our meta-analysis, including 2 high-quality studies and 10 low-quality studies. Three of the publications used correct RCT methods, and blinding was used correctly in all literatures. Two concealment methods were used appropriately; however, the remaining concealment methods used were unclear or improperly used. Among the selected publications, only Hong et al. [2017] (18) clearly explained the safety of the injections and the occurrence of adverse events; the other publications did not elaborate on these topics. The 12 publications specifically described the general information of the experimental and control groups.

This meta-analysis compared the performance status score effective rates, the objective curative effect effective rates, the TCM syndrome score changes, and the incidence of adverse digestive system reactions after chemotherapy in the experimental group treated with TCM combined with neoadjuvant chemotherapy, and the control group treated with chemotherapy alone. The efficiency of the performance status score was tested for heterogeneity $\left(\mathrm{Chi}^{2}=2.95, \mathrm{df}=5\right.$, $\mathrm{P}=0.71>0.1, \mathrm{I}^{2}=0 \%<50 \%$ ), and the combined effect-size test showed that $Z=3.36$. The combined effect size used a fixed-effects model (OR $=2.61,95 \% \mathrm{CI}, 1.49-4.58)$. A heterogeneity test was performed on the effective rate of the objective curative effect $\left(\mathrm{Chi}^{2}=1.04, \mathrm{df}=7, \mathrm{P}=0.99>0.1\right.$, $\left.\mathrm{I}^{2}=0 \%<50 \%\right)$. The combined effect-size test showed that $\mathrm{Z}=2.42$, OR $=2.00,95 \% \mathrm{CI}, 1.14-3.49$. A heterogeneity test was performed on the changes in the TCM syndrome scores $\left(\mathrm{I}^{2}=83 \%, \mathrm{P}<0.00001, \mathrm{MD}=8.84,95 \% \mathrm{CI}, 6.43-\right.$ 11.25). A random-effects model was used for the metaanalysis, and the difference between the experimental group and the control group was statistically significant $(\mathrm{P}<0.05)$. The incidence of adverse reactions in the digestive system after chemotherapy was tested for heterogeneity $\left(\mathrm{Chi}^{2}=1.15\right.$, $\left.\mathrm{df}=8, \mathrm{P}=1.00>0.1, \mathrm{I}^{2}=0 \%<50 \%\right)$. The combined effect-size test showed that $Z=1.68$, and the combined effect size used the fixed-effects model ( $\mathrm{OR}=0.04,95 \% \mathrm{CI},-0.01$ to 0.09 ). Rev Man 5.3 was used to produce the funnel chart of each observation index, and the circles of some publications were basically symmetrical with the midline, which indicates that the research accuracy was high and there was no bias in the publications. Consistent with the results of Tong et al. (19), we found that the combination of TCM and neoadjuvant chemotherapy had a notable advantage over the treatment of chemotherapy alone in improving performance status scores.

\section{Conclusions}

In this paper, a compound logic search method was used to identify 12 publications in which patients in the experimental group underwent neoadjuvant therapy combined with TCM and patients in the control group underwent chemotherapy alone. A meta-analysis was then conducted to explore the therapeutic effects of TCM combined with neoadjuvant therapy on breast cancer. The results of the meta-analysis confirmed that using TCM combined with neoadjuvant chemotherapy to treat breast cancer had obvious advantages over chemotherapy alone in terms of the objective curative effect, the performance status score effective rate, the TCM syndrome score change, and the incidence of gastrointestinal adverse reactions after chemotherapy. However, one limitation of this research is that the quality of the research objects was low, the heterogeneity was high, and the literature sample size included was small. Thus, the sample size needs to be expanded to conduct clinical RCTs in the later stage to provide verification of these results. In short, the results showed that TCM combined with neoadjuvant chemotherapy significantly improved the pathological symptoms of breast cancer patients.

\section{Acknowledgments}

Funding: None.

\section{Footnote}

Reporting Checklist: The authors have completed the PRISMA reporting checklist. Available at http://dx.doi. org/10.21037/gs-21-284

Conflicts of Interest: All authors have completed the ICMJE uniform disclosure form (available at http://dx.doi. org/10.21037/gs-21-284). The authors have no conflicts of interest to declare. 
Ethical Statement: The authors are accountable for all aspects of the work in ensuring that questions related to the accuracy or integrity of any part of the work are appropriately investigated and resolved.

Open Access Statement: This is an Open Access article distributed in accordance with the Creative Commons Attribution-NonCommercial-NoDerivs 4.0 International License (CC BY-NC-ND 4.0), which permits the noncommercial replication and distribution of the article with the strict proviso that no changes or edits are made and the original work is properly cited (including links to both the formal publication through the relevant DOI and the license). See: https://creativecommons.org/licenses/by-nc$\mathrm{nd} / 4.0 /$.

\section{References}

1. Liu CT, Chen YH, Huang YC, et al. Chemotherapy in conjunction with traditional Chinese medicine for survival of patients with early female breast cancer: protocol for a non-randomized, single center prospective cohort study. Trials 2019;20:741.

2. Huang CH, Chang HP, Su SY, et al. Traditional Chinese medicine is associated with a decreased risk of heart failure in breast cancer patients receiving doxorubicin treatment. J Ethnopharmacol 2019;229:15-21.

3. Sun Y, Liao M, He L, et al. Comparison of breastconserving surgery with mastectomy in locally advanced breast cancer after good response to neoadjuvant chemotherapy: A PRISMA-compliant systematic review and meta-analysis. Medicine (Baltimore) 2017;96:e8367.

4. Ji Y, Li S, Zhang X, et al. The prophylactic and therapeutic effects of moxibustion combined with traditional Chinese medicine decoction for treating chemotherapyinduced myelosuppression in early-stage breast cancer: study protocol for a randomized controlled trial. Trials 2020;21:844.

5. Liew AC, Peh KK, Tan BS, et al. Evaluation of chemotherapy-induced toxicity and health-related quality of life amongst early-stage breast cancer patients receiving Chinese herbal medicine in Malaysia. Support Care Cancer 2019;27:4515-24.

6. Li H, Yao L, Jin P, et al. MRI and PET/CT for evaluation of the pathological response to neoadjuvant chemotherapy in breast cancer: A systematic review and meta-analysis. Breast 2018;40:106-15.
7. Chen $\mathrm{Y}$, Wang L, Liu H, et al. PET Imaging on Dynamic Metabolic Changes after Combination Therapy of Paclitaxel and the Traditional Chinese Medicine in Breast Cancer-Bearing Mice. Mol Imaging Biol 2018;20:309-17.

8. Wang W, Xu L, Shen C. Effects of Traditional Chinese Medicine in Treatment of Breast Cancer Patients After Mastectomy: A Meta-Analysis. Cell Biochem Biophys 2015;71:1299-306.

9. Kim W, Lee WB, Lee JW, et al. Traditional herbal medicine as adjunctive therapy for breast cancer: A systematic review. Complement Ther Med 2015;23:626-32.

10. Liu Y, Pan T, Zou W, et al. Relationship between traditional Chinese medicine constitutional types with chemotherapy-induced nausea and vomiting in patients with breast cancer: an observational study. BMC Complement Altern Med 2016;16:451.

11. Meng H, Peng N, Yu M, et al. Treatment of triplenegative breast cancer with Chinese herbal medicine: A prospective cohort study protocol. Medicine (Baltimore) 2017;96:e8408.

12. Zhang Q, Gao X, Liu S, et al.Therapies for cognitive impairment in breast cancer survivors treated with chemotherapy: A protocol for systematic review. Medicine (Baltimore) 2020;99:e20092.

13. Yu X, Zhou S, Wang J, et al. Hormone replacement therapy and breast cancer survival: a systematic review and meta-analysis of observational studies. Breast Cancer 2017;24:643-57.

14. Lu Q, Dong L, Wu IHC, et al. The impact of an expressive writing intervention on quality of life among Chinese breast cancer patients undergoing chemotherapy. Support Care Cancer 2019;27:165-73.

15. Li Y, Wang J, Lin F, et al. A Methodology for Cancer Therapeutics by Systems Pharmacology-Based Analysis: A Case Study on Breast Cancer-Related Traditional Chinese Medicines. PLoS One 2017;12:e169363.

16. Zhang S, Guo N, Wan G, et al. pH and redox dualresponsive nanoparticles based on disulfide-containing poly( $\beta$-amino ester) for combining chemotherapy and COX-2 inhibitor to overcome drug resistance in breast cancer. J Nanobiotechnology 2019;17:109.

17. Su Y, Huang N, Chen D, et al. Successful in vivo hyperthermal therapy toward breast cancer by Chinese medicine shikonin-loaded thermosensitive micelle. Int $\mathrm{J}$ Nanomedicine 2017;12:4019-35.

18. Hong J, Chen X, Huang J, et al. Danggui Buxue Decoction, a Classical Formula of Traditional Chinese 
Medicine, Fails to Prevent Myelosuppression in Breast

Cancer Patients Treated With Adjuvant Chemotherapy: A Prospective Study. Integr Cancer Ther 2017;16:406-13.

19. Tong T, Pei C, Chen J, et al. Efficacy of Acupuncture Therapy for Chemotherapy-Related Cognitive

Cite this article as: Shi G, Yu D, Wu J, Liu Y, Huang R, Zhang CS. A systematic review and meta-analysis of traditional Chinese medicine with chemotherapy in breast cancer. Gland Surg 2021;10(5):1744-1755. doi: 10.21037/gs-21-284
Impairment in Breast Cancer Patients. Med Sci Monit 2018;24:2919-27.

(English Language Editor: L. Huleatt) 